\title{
Water Extraction from Air: Potential and Limitation
}

\author{
S. Gh. Etemad ${ }^{1}$, M. Haghshenas Fard ${ }^{1}$, B. Kruczek ${ }^{2}$, E. Jara-Morante ${ }^{3}$, J. Thibault ${ }^{2}$ \\ ${ }^{1}$ Department of Chemical Engineering \\ Isfahan University of Technology \\ Isfahan 84156-83111, Iran \\ ${ }^{2}$ Department of Chemical and Biological Engineering \\ University of Ottawa \\ Ottawa, Ontario, Canada K1N 6N5 \\ ${ }^{3}$ Facultad de Química e Ingeniería Química \\ Universidad Nacional Mayor de San Marcos \\ Lima, Peru
}

Collecting water from atmospheric air by condensation can help to partly alleviate water shortage in different regions of the world. Liquid water can be obtained when a surface exposed to moist air reaches a temperature that is below the dew point temperature. The surface can be cooled using a coolant or naturally by radiating to a clear night sky. This investigation examines the latter. The efficiency of this condensation process depends on numerous parameters such as the emissivity of the condensing surface, wind speed, air relative humidity, and air temperature. Through numerical simulations, the role of these parameters on the collector plate temperature and condensation rate was examined. Results show an interesting role of the wind speed on the resulting condensation rate on the plate. On the one hand, as the wind speed increases, the plate temperature increases, thereby resulting in a decrease in the driving force for condensation. On the other hand, the mass transfer coefficient for the transport of the water vapour from bulk air to the plate increases with the wind speed. The latter effect is stronger than the former one, therefore for the wind speeds for which the plate remains below the dew point temperature, the condensation rate increases with the wind speed. The effects of the relative humidity and the ambient air temperature were also examined to determine how they impact the ability of passive collectors to condense water. 